\title{
6 OPEN ACCESS \\ The Handover Toolbox: a knowledge exchange and training platform for improving patient care
}

\author{
Hendrik Drachsler, ${ }^{1}$ Wendy Kicken, ${ }^{1}$ Marcel van der Klink, ${ }^{1}$ Slavi Stoyanov, ${ }^{1}$ \\ Henny P A Boshuizen, ${ }^{1}$ Paul Barach, ${ }^{2,3,4}$
}

\begin{abstract}
${ }^{1}$ Centre for Learning Sciences and Technologies, Open University of the Netherlands, Heerlen, Limburg, The Netherlands ${ }^{2}$ Utrecht Medical Centre, Utrecht, The Netherlands ${ }^{3}$ University of Stavanger, Stavanger, Norway ${ }^{4}$ University College Cork, Ireland
\end{abstract}

Correspondence to Dr Hendrik Drachsler, Open University, Centre for Learning Sciences and Technologies,

Valkenburgerweg 177 , Heerlen, Limburg 6419 AT, The Netherlands; Hendrik. Drachsler@ou.nl

Accepted 27 September 2012

\section{$\mathrm{AB}$
$\mathrm{Ba}$
rem
Lim
apro
ha
the}

\section{ABSTRACT}

Background: Safe and effective patient handovers remain a global organisational and training challenge. Limited evidence supports available handover training programmes. Customisable training is a promising approach to improve the quality and sustainability of handover training and outcomes.

Objective: We present a Handover Toolbox designed in context of the European HANDOVER Project. The Toolbox aims to support physicians, nurses, individuals in health professions training, medical educators and handover experts by providing customised handover training tools for different clinical needs and contexts. Methods: The Handover Toolbox uses the Technology Enhanced Learning Design Process (TEL-DP), which encompasses user requirements analysis; writing personas; group concept mapping; analysis of suitable software; plus, minus, interesting rating; and usability testing. TEL-DP is aligned with participatory design approaches and ensures development occurs in close collaboration with, and engagement of, key stakeholders. Results: Application of TEL-DP confirmed that the ideal formats of handover training differs for practicing professionals versus individuals in health profession education programmes. Training experts from different countries differed in their views on the optimal content and delivery of training. Analysis of suitable software identified ready-to-use systems that provide required functionalities and can be further customised to users' needs. Interest rating and usability testing resulted in improved usability, navigation and uptake of the Handover Toolbox. Conclusions: The design of the Handover Toolbox was based on a carefully led stakeholder participatory design using the TEL-DP approach. The Toolbox supports a customisable learning approach that allows trainers to design training that addresses the specific information needs of the various target groups. We offer recommendations regarding the application of the Handover Toolbox to medical educators.

\section{INTRODUCTION}

Safe patient handovers require that accurate, reliable and relevant information is unambiguously communicated between healthcare providers. Improperly conducted handovers lead to wrong treatment, delays in medical diagnosis, life-threatening adverse events, patient complaints, increased healthcare expenditure, increased hospital length of stay and a range of other effects that impact on the health system. ${ }^{1-5}$ The WHO lists effective handovers as one of the High 5 patient safety initiatives. ${ }^{6}$ While training for handover skills is a promising approach to improve the quality of handovers, ${ }^{7-9}$ the need for handover training in medical education has not been clearly stated and present training methods have not been validated. Research has identified medical trainees' dissatisfaction with current handover practices as a result of a lack of clear policies and ineffective training. ${ }^{7}{ }^{10}$ Training in handover and patient safety is disjointed, lacks focus and there has been a paucity of research about formative or summative evaluation of present educational and training strategies. ${ }^{7}$ 11-13

Gaining insight into appropriate training strategies can thus provide guidelines for improving handover skills through training. However, training and learning alone are not sufficient to improve handovers. A supportive environment is also required to ensure the transfer of training and sustainability of the intervention. ${ }^{14}$ Thus, an effective solution to improve handovers will require a combination of the following: effective tools, protocols, methods (ie, checklists for standardised steps); interventions to change the culture around patient handovers (ie, role playing, simulation); and a supportive environment that allows the effective transfer of training into the workplace (ie, microsystem-based training and coaching). ${ }^{15}$ This framework must be maintained when searching for solutions to improve handovers. 
Handover procedures depend to a significant degree on context, handover practices and cultures, and the problems experienced vary across different institutions and European countries. ${ }^{1}{ }^{11} 16 \quad{ }^{16}$ Designing a one-size-fits-all training may not be practical or effective.

As part of the larger European HANDOVER Project, ${ }^{17}$ we developed a web-based knowledge exchange environment that enabled an online community of practice, ${ }^{18-20}$ which we named the Handover Toolbox (see http://www. handovertoolbox.eu). The Toolbox takes into account the diversity of needed solutions to improve handovers in varied patient care settings, and contains state of the art knowledge about standardised and ready to use tools to improve handovers; information on the content and format of handover training and ready to use training material; and guidelines on how efforts to improve handovers need to be sensitive to culture and organisational issues. Knowledge in these areas is continuously growing, yet the impact of interventions to improve handovers on patient care practices and patient safety is not clear. ${ }^{7} 9$ Thus, we developed a virtual environment that combines state-of-the-art information, contributions and cooperation from user groups, including their knowledge about the conditions for use, to improve the usability of the Toolbox.

The Handover Toolbox can be classified as an educational intervention using innovative teaching technologies, which aims to increase the knowledge and skills of target users to contribute to more effective handovers. We incorporated the experience of general practitioners, nurses, medical educational experts, experts on intervention mapping and experts on patient handover, using a 'Technology Enhanced Learning Design Process' (TEL-DP), which consists of six methods related to user participation in educational programme design. We first describe TEL-DP, then we describe the outcomes of its application and present the Handover Toolbox. We conclude with recommendations for the application of the Handover Toolbox to medical education in Europe and the role of TEL-DP in medical education.

\section{METHODS}

\section{The Technology Enhanced Learning Design Process}

TEL-DP was developed at the Centre for Learning Science and Technologies to provide customised technology-enhanced learning products for target domains such as logistics, industry and healthcare. It is similar to participatory design methods by including stakeholders in the design process. $^{21}$ Each of the six methods in TEL-DP provides input or guidance for the next step in the design process, resulting in the final design of a ready-to-use tool that is developed in close cooperation with target users (see figure 1). ${ }^{22}{ }^{23}$ Use of TEL-DP promotes user involvement to ensure a sound balance between the designers' vision and the needs and perspectives of the users. ${ }^{24}{ }^{25}$ This fundamental departure from traditional medical education practices initiates the engagement of end users at the start of the process. Formative and summative evaluation plays a prominent role, and the longitudinal process offers users and stakeholders insight into the latest solutions and assesses their appropriateness for the stakeholders' needs.

The application of TEL-DP to the development of the Handover Toolbox included user requirements analysis; writing personas; group concept mapping (GCM); analysis of suitable software; plus, minus, interesting (PMI) rating; and usability testing. We describe each of the methods and their outcomes on an aggregated level.

\section{User requirements analysis}

Our user requirements analysis was based on 35 semistructured interviews with medical trainers from the Netherlands, Poland and Spain who provided a list of requirements and issues about effective handovers. ${ }^{16} 26$ Stakeholders recommended an e-learning solution that can be used in role playing games and simulations to iteratively improve the handover process. They also emphasised the content and delivery of training needs to be as flexible as possible to enable professionals to be trained in the workplace, ${ }^{27}$ in addition to students and other learners. This suggested that one-size-fits-all training was not applicable, and there was benefit in a customisable learning approach through a toolbox that allows trainers to design their own programme tailored to the needs of trainees and their respective healthcare systems. ${ }^{9}$

\section{Writing personas}

The findings from the stakeholder interviews were used to create 'writing personas'. A persona is a synthesis of elements drawn from a large number of users who share common job roles, demographics, and user needs, which help designers to understand whom they are designing for. ${ }^{28}$ We created three different personas for providers in Spain, Poland and the Netherlands that sought to represent the average stakeholder in each country. The personas can be found online in the Handover Toolbox ${ }^{29}$

\section{Group concept mapping}

GCM is an element of TEL-DP that applies a structured participative approach to support the target users to achieve a consensus about particular issues. ${ }^{30}$ In the case of the Handover Toolbox, the issue of interest concerned criteria for effective and efficient handover 


\section{The Technology Enhanced Learning - Design Process}

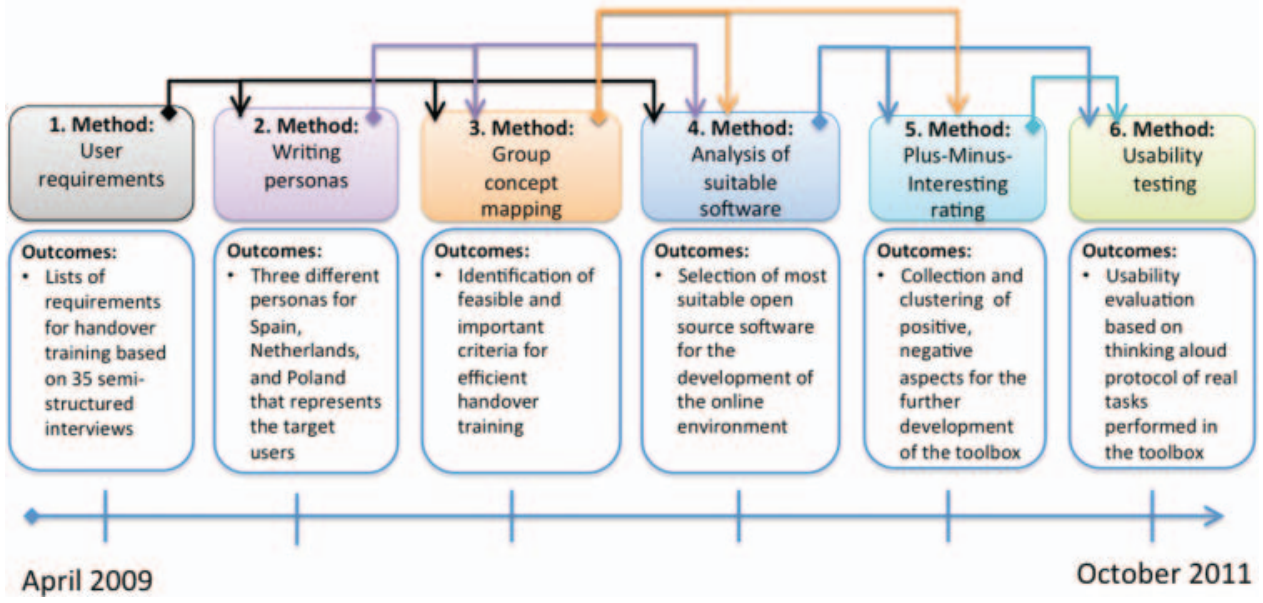

Figure 1 Overview of the Technology Enhanced Learning Design Process (TEL-DP) process in the FP7 HANDOVER Project to develop the Toolbox. The arrows above demonstrate the effects of a single method on the following step in the design process.

training. ${ }^{7} \mathrm{GCM}$ is a three-step approach that uses idea generation, sorting ideas and rating them with multidimensional scaling and hierarchical cluster analysis.

We first created a list of ideas from the interviews with the medical trainers. Fifteen experts then clustered and ranked these ideas according to importance and feasibility. The results were then discussed by the partners in the HANDOVER Project and the outcomes were taken into account in designing the Toolbox and its contents. A comprehensive overview of the outcomes of the GCM method applied in the HANDOVER Project is provided in another article. ${ }^{31}$

\section{Software selection}

The aim of the software selection process was to choose the web platform to support stakeholders' e-learning requirements. First, IT requirements were derived from the interviews and the writing personas to get a conceptual view on the collective IT needs of the stakeholder groups. This was followed by composing a use case diagram ${ }^{32}$ that demonstrated the desired functionalities of the system. Following this, a first version of the Handover Toolbox was created, which was based on the best candidate software system. This Toolbox was then further customised and enriched with content that was formally reviewed and 'certified' by a group of handover experts.

In the last two steps of TEL-DP, the prototype Toolbox was presented to different stakeholder groups to obtain their feedback and suggestions for improvement.

Plus, minus, interesting rating

The PMI rating method ${ }^{33}$ was used to collect feedback on the first version of the Handover Toolbox. The PMI rating was conducted at a stakeholder meeting of the HANDOVER Project during which 62 handover experts were given a demonstration of the possibilities of the Handover Toolbox. They were asked to write down statements on what they liked (marked with a plus sign) about the Handover Toolbox, what they disliked about it, or the objections they hold against it (marked with a minus sign), and interesting ideas that might help the designers to improve the Toolbox (marked with a capital I). The statements were then sorted on commonalities by three project team members using card sorting software. ${ }^{34}$

\section{Usability test}

We conducted a usability test to evaluate a refined version of the Handover Toolbox with three groups of training experts and medical professionals at the University Hospital in Barcelona, Spain ( $n=4)$, Karolinska Institute in Sweden $(\mathrm{n}=4)$, and the University Medical Centre, Utrecht, the Netherlands $(n=5)$. Participants were asked to perform three tasks with the toolbox (eg, search for information, join a group, and add a comment to existing information), while thinking out loud, sharing with the researchers what they are looking at, doing, and experiencing. They were interviewed, and also filled out a questionnaire consisting of 16 items with Likert scales and open-ended questions about their backgrounds and their perceptions on particular aspects of the Toolbox. The results of this contributed to improving the interface and Toolbox content.

\section{RESULTS}

In this section we present the final version of the Handover Toolbox, with a focus on its current 
functionalities and content. In total, 40 handover tools, 142 related files, 22 videos and 25 discussion topics have been created by the HANDOVER Project team and the Toolbox has 174 registered users to date.

\section{Handover Toolbox functionality}

The Handover Toolbox (http://www.handovertoolbox. eu) goes beyond serving as a website that offers static information to users. It offers various options for interaction (the ability to join an existing group or create new a new discussion group, and the ability to add files, post a blog or contribute a bookmark). The Toolbox is built around a proactive user concept, and offers users the option to define their own personal space and identify other individuals with similar goals or important expertise as a community of practice with a potentially huge member base. The navigation and functionality of the Toolbox is similar to social network sites; at the same time the Toolbox provides a trusted environment in which data and information in the system are owned and controlled by the users. It can be used as a place to exchange best practices and latest developments by experts; yet it can serve as a small-scale e-learning environment for individual trainers and their learners (see figure 2).

\section{Sign up for the handover toolbox}

When signing up for the Toolbox, users provide basic information that will become part of their personal profile within the Toolbox. After signing in, users wait for approval of their account by Toolbox administrator, as access is only provided to trustworthy people to protect the community from spam. After receiving approval, the new member can access the Toolbox and contact other members. The new user has the opportunity to join groups, create their own groups and comment on files.

\section{Groups}

The core element in the Toolbox is the notion of groups ${ }^{35}$ and the information related to various aspects of handovers is categorised and presented in relation to these groups. Each group has a theme and a group leader who formed the group and gathered the initial information. Six groups were initiated by the HANDOVER Project members, with themes derived from the information gathered by the interviews with medical trainers, the PMI rating and the usability test:

1. How to use the toolbox.

2. Protocols, checklists and other standardised tools to improve handover.

3. Communication skills, knowledge, awareness, and attitude.

4. Patient empowerment during handover practices.

5. External and organisational factors influencing the effectiveness of handover.

6. Handover training.

\section{Open and closed groups}

The default setting for groups is free access (open group) which allows visitors to see and download all information aggregated by the group. Any registered member of the Handover Toolbox can opt to become a member of a group. Membership allows possibilities for

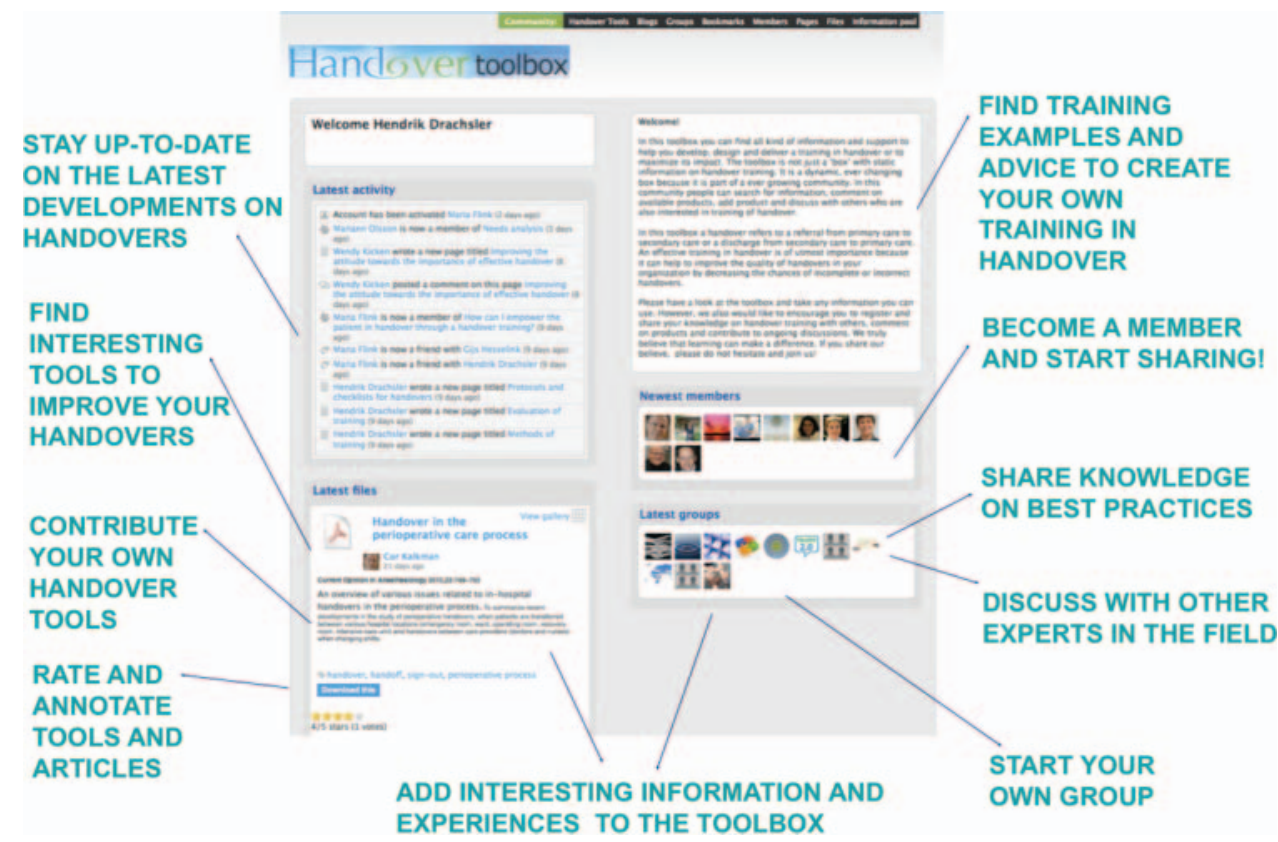

Figure 2 Functionalities of the HANDOVER Toolbox. 
active participation, including uploading one's own materials to the group. Some groups employ a restricted policy (closed group), in which the group leader decided who can become a member. This is particularly useful for educators who want to use the Handover Toolbox as a place where their trainees can find information and can discuss it with fellow trainees confidentially without this frank discourse hampered by the presence of outsiders. For these instances, a new group is created, trainees are invited to become members, and training assignments and other information are shared with them.

\section{Public access versus protected information}

A crucial aspect of the Handover Toolbox is the information management that allows users to specify who can edit or view a certain resource (a file, comment, blog, page or video). Individuals and groups who contribute content can make the following distinctions to make their material available in the Toolbox: public-the resource can be accessed by anyone; signed-in members-resources can only be accessed by members of the handover toolbox; only group members-only members of a particular group can access the content; and private-the content is visible to the individual user.

\section{Discussing, commenting, rating}

The user-generated content concept that underlies the Handover Toolbox values the active contribution of members. Users are encouraged to contribute to ongoing discussions or start new ones, and they have the opportunity to comment on all resources in the Toolbox. Users are also able to rate resources on a scale from one to five stars, which can be useful for others looking for an appropriate, 'tested' resource. The rating is a central instrument to highlight the most relevant information. It also helps to filter the high quality contributions that handover experts will consider for inclusion in the 'certified' content section.

\section{Adding files}

Registered users can always add files to their own personal profile. Once a file becomes part of the personal profile it can be linked and reused within the toolbox (in a discussion, group or blog posting). Members of groups are able to add files to the group's resources. This allows groups to develop a comprehensive collection of relevant files in a relatively short time.

\section{Contribute bookmarks}

The Toolbox has a powerful bookmarking instrument that enables its users to contribute relevant web pages as bookmarks directly to the Handover Toolbox. The so-called bookmarklet is a little button registered users can add to their browser. Whenever they discover an interesting webpage or resource they can click the new button (bookmarklet) in the browser, which brings up a web form. After filling out the form and pressing save, the website is directly stored in the Handover Toolbox. Depending on the information management settings, it may also be distributed to a specific group.

\section{Embed videos}

The Handover Toolbox allows the embedding of videos in any text area (blog, wiki page, etc) from a public video sharing site such as vimeo.org or youtube.com by copying and pasting the video link into a discussion, page or blog posting. User developed videos are incorporated by uploading them to a public video sharing site and integrating them into the Toolbox.

\section{'Certified' content in the Handover Tools section}

The property that all users can upload information to the Toolbox and start a new group was regarded as an appealing feature. At the same time, there is the danger that information may be added that is not reliable or may even be counterproductive for improving handovers. To assure the quality of the information, the Toolbox includes 'certified' content, called 'Handover Tools'. ${ }^{36}$ This provides users with the most relevant evidence-based tools, strategies and recommendations. The certified content in the Handover Tools section is organised around six categories: videotaped interviews with handover experts; content related to communication skills, knowledge awareness and attitude; certified protocols and handover checklists; content related to patient empowerment; information on organisational and external factors; and certified handover training materials. Information in the Handover Tools section can only be edited by experts in the Handover community. In the following section we will give a brief overview of the certified content section

\section{Interviews with handover experts on video}

The Tools section includes a collection of 22 interviews with patient handover experts from five countries. Interviews cover a broad range of topics, including a European research agenda around handover, the role of videotaping for reflection of handover processes, organisational culture in handover processes, and how process mapping can be used to understand the process of handover, among others. 
Communication skills, knowledge awareness and attitude

The HANDOVER Project advocates a combination of training in communication skills, improving knowledge of safe practices, increasing awareness to the potential for mistakes to happen and adapting attitudes accordingly, and this section is devoted to certified materials on these topics.

\section{Protocols and checklists for handover}

Current handover practices are highly variable. Standardisation of handover processes and content has the potential to improve safety by ensuring consistency in critical information exchanges. Tools in this section are divided into four categories, relevant to different types of handovers: intra-organisational handovers (handover within the hospital or primary care service; medication handovers; content of handovers; and materials related to standardising handover processes.

\section{Patient empowerment}

This section recognises that patients and their families are at the centre of the handover process and can play an important role in influencing the quality of handovers. ${ }^{37}$ It includes approaches for improving communication around handovers between patients and healthcare professionals, and was informed by the project's patient advisory group.

\section{Organisational and external factors}

This section of Handover Tools includes tools and strategies for describing and assessing organisational and external factors that influence handovers. Tools in this section aid in describing and assessing the features that influence handover implementation, and result from state-of-the-art research.

\section{A generic handover training programme}

The Handover Tools section is a set of 'certified' tools that range from a description of how to apply process mapping to tutoring strategies for effective instruction. They comprise building blocks for handover training programmes and can be assembled using the ADDIE model. ${ }^{38}$ ADDIE is a generic process used by instructional designers and training developers that includes analysis, design, development, implementation, and evaluation. It offers a dynamic, flexible set of building blocks that can be selected and combined by training designers to build customised training for certain demands in handover.

There are three limitations of the Handover Toolbox that need to be taken into account and should be addressed in future research. While we are confident that we addressed the requirements expressed by the target users through the TEL-DP approach, some senior healthcare professionals were not used to social network navigation concepts, which may create a barrier to full adoption of tools and concepts from the Toolbox by all professionals. Carefully monitored test implementation of the Toolbox at specific target institutions will create knowledge about how to embed this resource into local training approaches. We plan to provide access to tools on mobile (handheld) devices, which will assist in guiding handovers during training situations and in real life implementation. Second, our generic training programme requires further development as it appears to be too general for practical application. Training will need to be expanded in the areas of medical knowledge and clinical decision-making skills related to the handover to ensure that the 'right' information is communicated and sufficiently understood by the other caregivers of the patient. ${ }^{39}$

Finally, while we believe that the Toolbox will provide medical educators with state-of-the-art e-learning tools and content, our study did not collect empirical evidence that use of the Toolbox will make handovers more effective. Large-scale testing is needed to study the impact of the Toolbox on handover skills for students and healthcare professionals in the workplace, and the effect on improved safety and clinical outcomes.

\section{CONCLUSIONS}

While the literature mentions the need for handover training, there are no comprehensive accessible training programme tools. The Handover Toolbox used active involvement of stakeholders to address this void, by providing online resources and a community to support educators in the design and delivery of customised handover training. The Toolbox can also facilitate research on the effectiveness of handover training by disseminating generic training to a sufficient number of groups that produce data which will offer further insight into the effectiveness of training and its impact on patient care safety and quality. The Toolbox can support a shared vision and a training approach to support healthcare professionals who face an increasing amount of handovers at their workplace.

We see the Toolbox as a first step towards improving handover training and offering customisable solutions for medical educators. The emphasis should be on an adaptable, flexible training approach that enhances the potential for adoption and engagement by busy clinicians. Implementation of this training was only partly achieved within the European HANDOVER Project, and a follow-up project has begun that will implement the Toolbox in three university hospitals in Germany, Spain and Ireland. ${ }^{39}$ 
Contributors HD: initiated the article and contributed to acquisition of data, analysis and interpretation of data, drafting the article (all sections), revising it critically, and final approval of the version to be published. WK: substantial contributions to conception and design of the article, acquisition of data, analysis and interpretation of data, drafting the article (method and Toolbox content), and final approval of the version to be published. MvdK: substantial contributions to conception and design, acquisition of data, analysis and interpretation of data, drafting the article (method and Toolbox functionality), revising it critically, and final approval of the version to be published. SS: substantial contributions to conception and design, interpretation of data, contribution to the article (method section), revising the article critically, and final approval. HB: substantial contributions to conception and design, interpretation of data, revising the article critically, and final approval. PB: substantial contributions to revising the article critically, drafting the article (all sections) and final approval.

Funding European Union, the Framework Programme of the European Commission FP7-HEALTH-F2-2008-223409.

\section{Competing interests None.}

Ethics approval Participants contributed of their own free will.

Provenance and peer reviewed Not commissioned; externally peer reviewed.

Open Access This is an Open Access article distributed in accordance with the Creative Commons Attribution Non Commercial (CC BY-NC 3.0) license, which permits others to distribute, remix, adapt, build upon this work noncommercially, and license their derivative works on different terms, provided the original work is properly cited and the use is non-commercial. See: http:// creativecommons.org/licenses/by-nc/3.0/

\section{REFERENCES}

1. Joint Commission on Accreditation of Healthcare Organizations. Root causes of sentinel events. http://www.jointcommission.org/ SentinelEvents/Statistics/ (accessed 10 Sep 2012).

2. Cohen MD, Hilligoss PB. The published literature on handoffs in hospitals: deficiencies identified in an extensive review. Qual Saf Health Care 2010;19:493-7.

3. Philibert I. Use of strategies from high reliability organisations to the patient handoff by resident physicians: practical implications. Qual Saf Health Care 2009;18:261-6.

4. Arora V, Johnson J, Lovinger D, et al. Communication failures in patient sign-out and suggestions for improvement: a critical incident analysis. Qual Saf Health Care 2005;14:401-7.

5. Gandhi TK. Fumbled handoffs: one dropped ball after another. Ann Intern Med 2005;142:352-8.

6. Joint Commission for Patient Safety and High 5 s Initiative. http://www. jointcommissioninternational.org/WHO-Collaborating-Centre-forPatient-Safety-Solutions/ (accessed 10 Sep 2012).

7. Gordon M, Findley R. Educational interventions to improve handover in health care: a systematic review. Med Education 2011;45:1081-9.

8. Arora VM, Johnson JK, Meltzer DO, et al. A theoretical framework and competency based approach to improving handoffs. Qual Saf Health Care 2008;17:11-14.

9. Wohlauer MV, Arora VM, Horwitz LI, et al. The patient handoff: a comprehensive curricular blueprint for resident education to improve continuity of care. AAMC Acad Med J Assoc Am Med Coll 2012;87:411-18.

10. Gordon M. Handover in paediatrics. Junior perceptions of current practice in the northwest region. Arch Dis Child 2010;95(Suppl 1):69.

11. Johnson J, Barach P. Patient care handovers: what it will take to ensure quality and safety during times of transition. Med J Aust 2009;190:110-12.

12. Reisenberg LA, Jaeger J, Padmore JS. Residents' and attending physicians' hand-offs: a systematic review of the literature. Acad Med 2009;84:1775-87.

13. Jeffcott SA, Evans SM, Cameron PA, et al. Improving measurement in clinical handover. Qual Saf Health Care 2009;18:272-7.
14. Entin E, Lei $F$, Barach P. Teamwork skills training for patient safety in the peri-operative environment: a research agenda. Surg Innov 2006;3:3-13.

15. Australian Medical Association. Safe Handover: Safe PatientsGuidance on Clinical Handover for Clinicians and Managers, 2006 http://www.ama.com.au/node/4064

16. Kicken W, Van der Klink M, Barach P. Handover training: does one size fit all? The merits of mass customization. BMJ Qual Saf, In press.

17. Barach $\mathrm{P}$, Gademan $\mathrm{P}$, Kalkman $\mathrm{C}$, et al. Identify basic elements of effective communication. Utrecht: The European HANDOVER Research Collaborative, 2010.

18. Fuller A, Hodkinson $\mathrm{H}$, Hodkinson $\mathrm{P}$, et al. Learning as peripherals participation in communities of practice: a reassessment of key concepts in workplace learning. Br Educ Res J 2002;31:49-68.

19. Brathwaite J, Runciman WB, Merry AF. Towards safer, better healthcare: harnessing the natural properties of complex sociotechnical Systems. Qual Saf Health Care 2009;18:37-41.

20. Ranmuthugala G, Cunningham FC, Plumb JJ, et al. A realist evaluation of the role of communities of practice in changing healthcare practice. Implement Sci 2011;6:49

21. Schuler D, Namioka A. Participatory Design: Principles and Practices. Mahwah, NJ: Lawrence Erlbaum Associates, 1993.

22. Scandurra I, Hägglund M, Koch S. From user needs to system specifications: multi-disciplinary thematic seminars as a collaborative design method for development of health information systems. $J$ Biomed Inform 2008;41:557-69.

23. Timpka T, Nyce JM. Dilemmas at a primary health care center: a baseline study for computer-supported cooperative health care work. Methods Inform Med 1992;31:204-9.

24. Stoyanov S, Kicken W, Boon J, et al. Ontwerpbenaderingen voor leernetwerken [Design approaches for learning networks]. In: Sloep PB, van der Klink M, Brouns F, van Bruggen J, Didderen W. Leernetwerken; Kennisdeling, kennisontwikkeling en de leerprocessen. Houten, The Netherlands: Bohn, Stafleu, Van Loghum, 2011:71-86.

25. Technology Enhanced Learning Design Process (TEL-DP), a more detailed description. http://dspace.ou.nl/handle/1820/3979 (accessed 28 Sep 2012)

26. European HANDOVER Project. http://handover.cmj.org.pl/upload/ library/m1b8hmo6d0hh9fiybvx8w.pdf (accessed 28 Sep 2012).

27. Marsick V. Informal strategic learning in the workplace. In: Streumer JN. Work-related Learning. Dordrecht: Springer, 2006:51-69.

28. Pruitt J, Grudin J. Personas: practice and theory. In: Proceedings of the 2003 Conference on Designing for User Experiences (DUX '03). New York: ACM, 2003:1-15. doi:10.1145/997078.997089

29. HANDOVER Toolbox. http://handover.ou.nl/pg/blog/read/8392/ writing-personas-representative-users-for-the-toolbox-stakeholders (accessed 28 Sep 2012).

30. Trochim W. An introduction to concept mapping for planning and evaluation. Eval Program Plann 1989;12:1-16.

31. Stoyanov S, Boshuizen $\mathrm{H}$, Groene $\mathrm{O}$, et al. Mapping and assessing clinical handover training interventions. BMJ Qual Saf, doi:10.1136/ bmjqs-2012-001169.

32. Denney R. Succeeding with Use Cases: Working Smart to Deliver Quality. Reading, MA: Addison-Wesley, 2005.

33. De Bono E. Serious creativity: using the power of lateral thinking to create new ideas. New York: Harper Business, 1992.

34. Websort Card Sorting Software, City, state/nation. http://websort.net (accessed 14 May 2012).

35. Handover Toolbox, Groups. http://handover.ou.nl/pg/groups (accessed 28 Sep 2012)

36. Handover Tools Section. http://handover.ou.nl/pg/pages/view/3587/ (accessed 28 Sep 2012).

37. Flink M, Hesselink G, Pijnenborg L, et al. The key actor-a qualitative study of patient participation in the handover process in Europe. BMJ Qual Saf, In press.

38. Molenda M. In search of the elusive ADDIE model. Perform Improve 2003;42:34-7.

39. patient-project.eu. http://www.patient-project.eu (accessed 28 Sep 2012). 